\title{
p53, cellular proliferation, and apoptosis- related factors in thymic neuroendocrine tumors
}

\author{
Anthony A Gal ${ }^{1}$, Mary N Sheppard ${ }^{2}$, John DL Nolen ${ }^{1}$ and Cynthia Cohen ${ }^{1}$ \\ ${ }^{1}$ Department of Pathology and Laboratory Medicine, Emory University School of Medicine, Atlanta, GA, USA \\ and ${ }^{2}$ Department of Pathology, Royal Brompton National Heart and Lung Hospital, London, UK
}

\begin{abstract}
Thymic neuroendocrine tumors are biologically aggressive neoplasms with extensive local invasion and high mortality. Although various markers of cellular proliferation and apoptosis have correlated with degrees of tumor differentiation in pulmonary neuroendocrine neoplasms, they have not been systematically studied in thymic neuroendocrine tumors. We immunostained 21 cases of thymic neuroendocrine tumors for p53, MIB-1, and the apoptosis-related markers Bcl-2, Bcl-x, and Bax. By histological classification the cases were low-grade (nine cases), intermediate-grade (eight cases), and high-grade (four cases) thymic neuroendocrine tumors. p53 was expressed in five cases: $1 / 9$ low grade, $3 / 8$ intermediate grade, and 2/4 high grade. The mean cellular proliferation (MIB-1) was 7.1\% (range 2-12\%) in low-grade thymic neuroendocrine tumors, $6.1 \%$ (range 2-15\%) in intermediate-grade thymic neuroendocrine tumors, and $34.2 \%$ (range 2-80\%) in high-grade thymic neuroendocrine tumors. Bcl-2 was expressed in 16 cases: 7/9 low grade, 5/8 intermediate grade, and 4/4 high grade. Bcl-x was expressed in 16 cases: $7 / 9$ low grade, 6/8 intermediate grade, and 3/4 high grade. Bax was expressed in 13 cases: 5/9 low grade, 4/8 intermediate grade, and 4/4 high grade. The presence of mutant p53 in the tumor was associated with a statistically significant decreased mean survival $(P<0.05)$. In contrast, either by positive or negative staining or by the score technique (staining intensity $\times$ percentage of cells staining), the presence of $\mathrm{Bcl}-\mathrm{x}$ was associated with an increased mean survival $(P<0.05)$. Finally, a Bcl-x : Bax ratio $\geq 1$ was also associated with an increased mean survival, as compared to a $\mathrm{Bcl-x}$ : Bax ratio $\geq 1(P<0.05)$. Our study shows that p53 expression and certain apoptosis markers correlate with survival. The expression of these markers may account for differences in biological behavior.
\end{abstract}

Modern Pathology (2004) 17, 33-39, advance online publication, 19 November 2003; doi:10.1038/modpathol.3800009

Keywords: thymus; mediastinum; carcinoid tumor; neuroendocrine carcinoma; apoptosis; p53; Bcl-2; Bcl-x; Bax; MIB-1 cellular proliferation

In contrast to those in the lung, thymic neuroendocrine tumors are rare aggressive tumors that frequently present at an advanced stage, invade lung or other mediastinal structures, and often metastasize to distant sites. ${ }^{1-3}$ On occasion, thymic neuroendocrine tumors secrete adrenocorticotrophic hormone leading to Cushing's syndrome. ${ }^{4}$ Rarely, these tumors can be associated with multiple endocrine neoplasia syndromes. ${ }^{5}$

Although the term 'thymic carcinoid tumor' has been used in the past, this designation may not be applicable for all thymic neuroendocrine tumors,

Correspondence: AA Gal, Department of Pathology and Laboratory Medicine, Emory University Hospital, H-171, 1364 Clifton Road, N.E., Atlanta, GA 30322, USA.

E-mail: agal@emory.edu

Received 11 April 2003; revised 24 July 2003; accepted 22 September 2003; published online 19 November 2003 since there is considerable morphological diversity ${ }^{6-8}$ Recently, Moran and Suster ${ }^{9}$ have proposed a three-tiered classification of low-, intermediate-, and high-grade neuroendocrine carcinomas of the thymus. However, there is conflicting data as to whether the degree of tumor differentiation correlates with survival. ${ }^{2}$ Thus, histology alone cannot predict the potential aggressive behavior of thymic neuroendocrine tumors.

Within the past decade, many studies have investigated the role of cellular proliferation and apoptosis in tumor cell biology. ${ }^{10-12}$ Altered or enhanced cellular proliferation is seen in various tumors and is influenced by a myriad of factors. Apoptosis requires a complex coordinated pathway involving various cell signaling and proteins through a cascade of events leading to cell death. The role of cellular proliferation and apoptosisrelated markers has not been systematically ex- 
plored in thymic neuroendocrine tumors. In this study, we examined various thymic neuroendocrine tumors for p53, MIB-1 (cellular proliferation), and Bcl-2, Bcl-x (antiapoptosis markers) and Bax (proapoptosis marker) to possibly explain differences in tumor cell biology.

\section{Materials and methods}

We retrieved 21 thymic neuroendocrine tumors from the pathology files at Emory University Hospital (Atlanta, GA, USA) (eight cases) and Royal Brompton Hospital (London, England) (13 cases). For inclusion into the study, each case demonstrated the morphological features of a thymic neuroendocrine tumor and showed expression of one or two neuroendocrine markers (ie chromogranin and synaptophysin). ${ }^{1,7,8}$ The cases were classified into low-, intermediate-, and high-grade neuroendocrine tumors in accordance with the histopathological criteria set forth by Moran and Suster. ${ }^{9}$ Additional clinical and pathological data for most of the cases previously have been reported in publications from both institutions. ${ }^{2,13,14}$ Clinical follow-up was obtained in each case; duration of survival and outcome were recorded. The cases were staged according to the criteria proposed by Masaoka et $a l:{ }^{15}$ Stage 1: macroscopically completely encapsulated and microscopically no capsular invasion; Stage 2a: macroscopic invasion into surrounding fatty tissue mediastinal pleura or both; Stage 2b: microscopic invasion into capsule; Stage 3: macroscopic invasion into a neighboring organ (pericardium, great vessel, lung, etc); Stage 4a: pleural and/ or pericardial dissemination; Stage 4b: lymphogenous and/or hematogenous metastasis. A lung primary tumor was excluded in all patients.

In each case, either an adequate paraffin block or unstained tissue sections were available. Immunohistochemistry for p53, Ki-67 (MIB-1), and apoptosis-related markers (Bcl-2, Bcl-x, and Bax) was performed using a steam heat antigen retrieval method with an avidin-biotin enzyme complex kit (LSAB2, DAKO Corp., Carpinteria, CA, USA) using an automated Dako-stainer (DAKO) (Table 1). Negative controls had primary antibody replaced by buffer. Polyclonal antibodies served as isotypespecific controls for each other.

Formalin-fixed, paraffin-embedded tissue sections $(5 \mu \mathrm{m})$ with thymic neuroendocrine tumors were deparaffinized and rehydrated, then steamed in citrate buffer ( $\mathrm{pH} \mathrm{6)}$ for $20 \mathrm{~min}$, and cooled for $10 \mathrm{~min}$ before immunostaining. All sections were exposed to $3 \%$ hydrogen peroxide for $5 \mathrm{~min}$, primary antibody for $25 \mathrm{~min}$, biotinylated secondary linking antibody for $25 \mathrm{~min}$, streptavidin enzyme complex for $25 \mathrm{~min}$, diaminobenzidine as chromogen for $5 \mathrm{~min}$, and hematoxylin as counterstain for $1 \mathrm{~min}$. These incubations were performed at room temperature. Between incubations, sections were washed with Tris-buffered saline. Coverslipping was performed using the Tissue-Tech SCA (Sakura Finetek USA, Inc.) automated coverslipper.

p53 nuclear and Bcl-2, Bcl-x, and Bax nuclear and cytoplasmic immunoreactivity were semiquantitively graded according to the intensity of staining $(0-3+)$ and percentage of cells staining, in a blinded fashion, by one of us (CC). The staining results were considered to be positive when at least $10 \%$ of tumor cells demonstrated immunoreactivity with a specific antibody. In addition to the analysis of strict positive or negative staining, a score was calculated by multiplying the staining intensity by the staining percentage. ${ }^{16}$ Cellular proliferation related to MIB-1 staining was assessed by manually counting the number of MIB-1 nuclear-positive cells in a 1000 cell count, converted to a percentage of total cells.

\section{Statistical analyses}

Of the 21 cases studied, 18 had complete follow-up data amenable to both survival and correlational analyses. The data were analyzed using SAS version 8.2 on a Windows NT platform. Both categorical and survival analyses were performed on the data, and statistical significance was set at a $P$-value of less than 0.05. Survival times were recorded from the date of diagnosis to the termination of follow-up. Patients who were alive or alive with disease were considered as 'censored', while death was considered an 'event'.

The association of positive or negative p53 and Bcl-2, Bcl-x, and Bax staining was correlated with tumor stage and histological grade using Fisher's exact test due to the small sample size and subsequent small cell sizes. The effect of the various factors on survival was assessed using the KaplanMeier method, with the log-rank test employed to test significance due to its bias towards the detection of differences between later survival times. MIB-1 staining was used as a marker of cellular prolifera-

Table 1 Antibody sources

\begin{tabular}{llllll}
\hline Antibody & Clone & Type & Dilution & Source & Positive control \\
\hline p53 & DO-7 & Monoclonal & $1: 20$ & Novacastra, NewCastle upon Tyne, Great Britian & Colonic adenocarcinoma \\
Ki67 & MIB-1 & Monoclonal & $1: 50$ & Immunotech, Hileah, FL & Tonsil \\
Bcl-2 & Oncl2+ & Monoclonal & $1: 80$ & Dako, Carpinteria, CA & Tonsil \\
Bcl-x & - & Polyclonal & $1: 1500$ & PharMingen, San Diego, CA & Tonsil \\
Bax & - & Polyclonal & $1: 1500$ & PharMingen, San Diego, CA & Tonsil
\end{tabular}


tion, and the relationship of MIB-1 with survival was assessed both with the actual MIB-1 score (0$100 \%$ ) and with a single cutpoint of $10 \%$. Also, the inter-relationships of the various factors with each other were assessed using Fisher's exact test.

A score, calculated by multiplying the staining intensity by the staining percentage, was also used for categorical and survival analyses. ${ }^{16}$ This analysis was used to facilitate a methodological comparison with similar published works. This score was also used to determine the Bcl-2:Bax and Bcl-x:Bax ratios; a ratio $\geq 1$ was treated as a positive score.

\section{Results}

The clinical, pathological, and immunohistochemical features are summarized in Table 2. The majority of patients presented at an advanced clinical stage (Stage 3 (four patients) and Stage 4 (10 patients)), whereas the remainder were early stage (Stage 1 (two patients) and Stage 2 (two patients)), or unknown (three patients). At the conclusion of the study, three patients had no evidence of disease, four patients were alive with disease, 11 patients had died with disease, and three patients were of an unknown status. The duration of follow-up ranged from 1 to 80 months, with an overall median survival of 58 months.

The cases were classified as low-grade (nine cases), intermediate-grade (eight cases), and highgrade (four cases) thymic neuroendocrine tumors (Figure 1). p53 immunoreactivity was more frequently present in intermediate (3/8) and high-grade thymic neuroendocrine tumors $(3 / 4)$, but was un- common in low-grade thymic neuroendocrine tumors (2/9) (Figure 2). The intensity of nuclear staining was moderate to strong in most cases. Moreover, the majority of high-grade thymic neuroendocrine tumors exhibited frequent nuclear staining and higher scores, as opposed to scattered immunopositive cells and lower scores in low- and intermediate-grade thymic neuroendocrine tumors.

High MIB-1 staining tended to occur more frequently in tumors of high histological grade, although many tumors showed evidence of enhanced cellular proliferation (Figure 3). The mean proliferation rates of the low- and intermediategrade thymic neuroendocrine tumors were comparable-7.1\% (range $2-12 \%$ ) vs $6.1 \%$ (range $2-15 \%$ ), respectively-whereas high-grade thymic neuroendocrine tumors had a high proliferation rate of $34.2 \%$ (range $2-80 \%$ ).

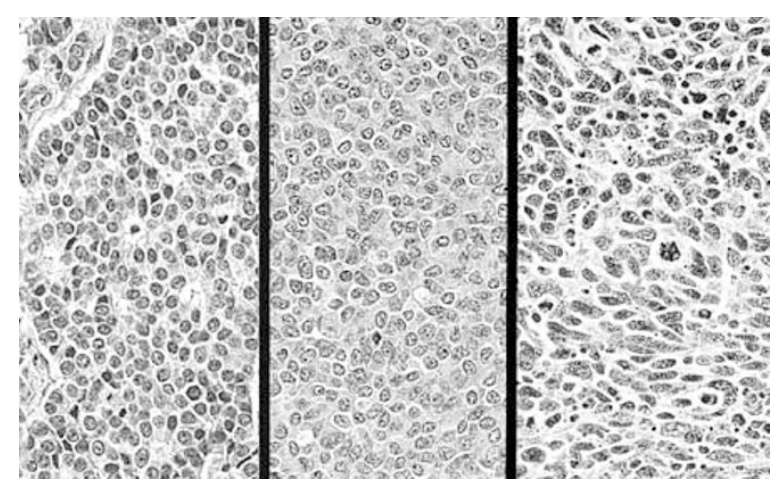

Figure 1 Low-grade (left), intermediate-grade (middle), and highgrade (right) neuroendocrine tumors of the thymus, hematoxylin, and eosin, $\times 80$.

Table 2 Clinical, histopathological, and immunohistochemical findings in neuroendocrine tumors of the thymus

\begin{tabular}{|c|c|c|c|c|c|c|c|c|c|}
\hline Patient & Stage & Follow-up & $\begin{array}{l}\text { Survival } \\
\text { (months) }\end{array}$ & $\begin{array}{l}\text { Histological } \\
\text { grade }\end{array}$ & p53 & $B C L-2$ & $B C L-X$ & $B A X$ & MIB-1 \% \\
\hline 1 & 3 & ANED & 58 & Low & $3+10 \%$ & $3+60 \%$ & $2+90 \%$ & $1+50 \%$ & 10 \\
\hline 2 & 4 & AWD & 48 & Low & $2+10 \%$ & 0 & $2+60 \%$ & 0 & $<2$ \\
\hline 3 & 2 & DWD & 1 & Low & 0 & $3+90 \%$ & $2+90 \%$ & $3+90 \%$ & 10 \\
\hline 4 & 4 & DWD & 22 & Low & 0 & $3+90 \%$ & 0 & 0 & 5 \\
\hline 5 & 2 & DWD & 78 & Low & 0 & $1+20 \%$ & $1+90 \%$ & 0 & 5 \\
\hline 6 & 1 & ANED & 80 & Low & 0 & $3+90 \%$ & $2+90 \%$ & $2+90 \%$ & 12 \\
\hline 7 & N/A & N/A & N/A & Low & 0 & $2+40 \%$ & 0 & 0 & 5 \\
\hline 8 & N/A & N/A & N/A & Low & 0 & 0 & $3+90 \%$ & $2+70 \%$ & 5 \\
\hline 9 & 3 & ANED & 74 & Low & 0 & $3+30 \%$ & $3+90 \%$ & $3+90 \%$ & 10 \\
\hline 10 & 3 & DWD & 45 & Intermediate & 0 & 0 & 0 & 0 & $<2$ \\
\hline 11 & 4 & DWD & 59 & Intermediate & $3+10 \%$ & 0 & $2+100 \%$ & 0 & 2 \\
\hline 12 & 4 & DWD & 33 & Intermediate & $3+15 \%$ & $3+90 \%$ & $2+90 \%$ & $3+90 \%$ & 2 \\
\hline 13 & 4 & AWD & 50 & Intermediate & $3+10 \%$ & 0 & $3+90 \%$ & $2+5 \%$ & 5 \\
\hline 14 & N/A & N/A & N/A & Intermediate & 0 & $2+50 \%$ & $1+90 \%$ & 0 & 5 \\
\hline 15 & 3 & DWD & 58 & Intermediate & 0 & $3+90 \%$ & 0 & $2+40 \%$ & 10 \\
\hline 16 & 4 & AWD & 60 & Intermediate & 0 & 0 & $3+90 \%$ & $3+90 \%$ & 15 \\
\hline 17 & 1 & AWD & 60 & Intermediate & 0 & $2+80 \%$ & $2+90 \%$ & $3+90 \%$ & 8 \\
\hline 18 & 4 & DWD & 47 & High & $3+30 \%$ & $3+95 \%$ & $3+80 \%$ & $1+10 \%$ & 5 \\
\hline 19 & 4 & DWD & 52 & High & $1+10 \%$ & $2+90 \%$ & $2+90 \%$ & $2+90 \%$ & 2 \\
\hline 20 & 4 & DWD & 26 & High & $3+50 \%$ & $2+70 \%$ & 0 & $2+90 \%$ & 80 \\
\hline 21 & 4 & DWD & 4 & High & 0 & $2+10 \%$ & $3+80 \%$ & $3+90 \%$ & 50 \\
\hline
\end{tabular}

ANED, alive with no evidence of disease; AWD, alive with disease; DWD, died with disease; N/A, not available. 
36

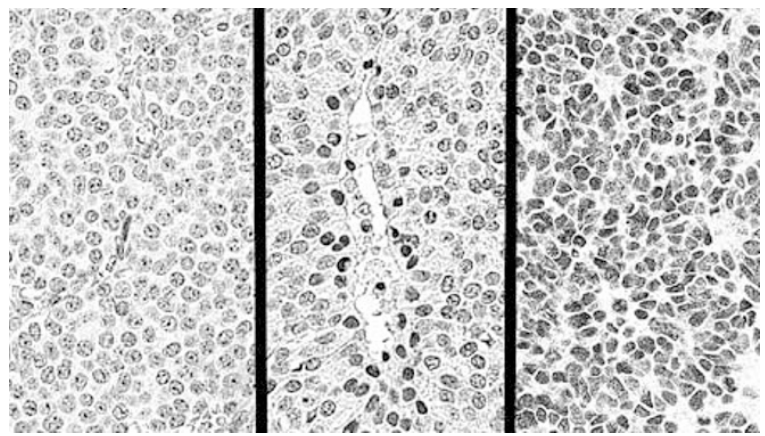

Figure 2 p53 immunostaining in low-grade (left), intermediategrade (middle), and high-grade (right) thymic neuroendocrine tumors, $\times 80$.

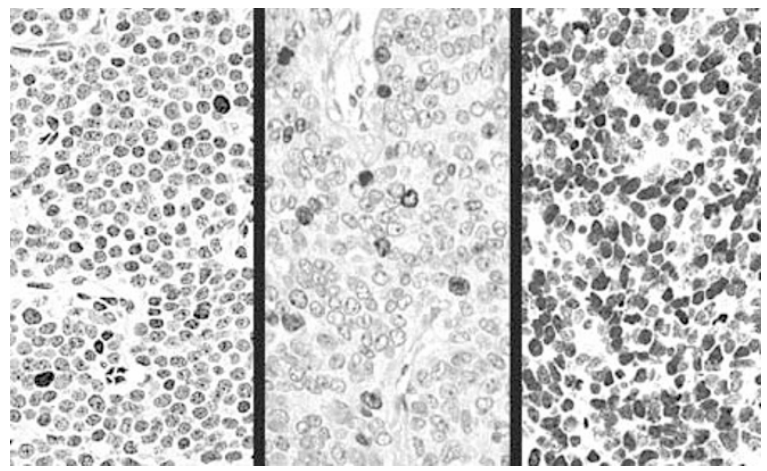

Figure 3 MIB-1 immunostaining in low-grade (left), intermediategrade (middle), and high-grade (right) thymic neuroendocrine tumors, $\times 80$.

There was variable but frequent expression of apoptosis markers in thymic neuroendocrine tumors: Bcl-2 in 16 cases (7/9 low grade, 4/8 intermediate grade, and 4/4 high grade), Bcl-x in 16 cases (7/9 low grade, 6/8 intermediate grade, and $3 / 4$ high grade), and Bax in 13 cases (5/9 low grade, 4/8 intermediate grade, and 4/4 high grade) (Figure 4). The markers exhibited finely granular cytoplasmic and occasionally nuclear or perinuclear immunoreactivity. Within a specific histological group, however, there was considerable variation in the staining intensity, frequency of immunopositive cells, and scores (Table 2).

The survival analyses yielded four significant associations. First, the presence of p53 in the tumor was associated with a statistically significant decreased mean survival (35 vs 56 months) $(P<0.05)$. Second, a statistically significant increased mean survival (58 vs 38 months) was seen with the presence of Bcl-x in the tumor. $(P<0.05)$ if scores were used in lieu of the $10 \%$ positive cutoff, Bcl-x was again found to have a statistically significant increased mean survival (58 vs 38 months) $(P<0.05)$. Finally, the Bcl-x:Bax ratio score $\geq 1$ was found to have a statistically significant increased mean survival (52 vs 43 months) $(P<0.05)$.
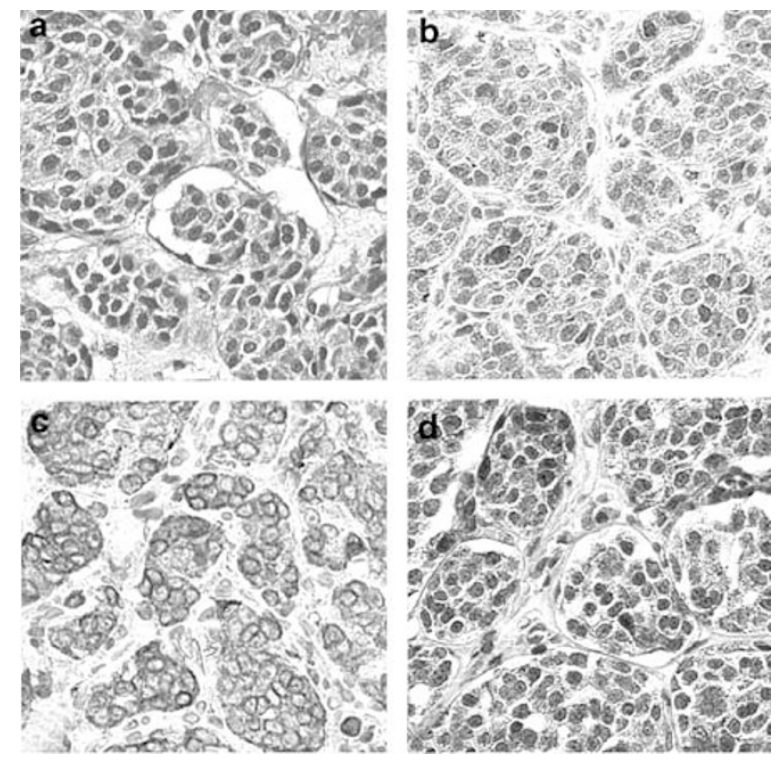

Figure 4 Apoptosis markers in thymic neuroendocrine tumors: hematoxylin and eosin (a), Bax (b), Bcl-2 (c), Bcl-x (d), $\times 80$.

For the categorical analyses, none of the individual factors were found to have a statistically significant association with either the grade or stage of the tumor. This lack of association persisted even with the use of scores applied to the staining patterns in the data. There were no statistically significant inter-relationships between individual factors. The correlation of MIB-1 with histology, stage, or survival was also not significant.

\section{Discussion}

There is considerable evidence to suggest that mutant p53 expression, increased cellular proliferation, and altered apoptosis are critical mechanisms that relate to differences in tumor biology and behavior. In thymomas, several studies have shown minimal mutant p53 expression, low proliferation rates, and variable expression of pro- and antiapoptosis markers. ${ }^{17-23}$ Thymic carcinomas, in contrast, generally have shown increased mutant p53 expression, higher proliferation rates, and a tendency to express Bcl-2. ${ }^{16,19-25}$ Although many of the previous studies have focused on non-neuroendocrine thymic neoplasms, there has been very little attention regarding these factors in thymic neuroendocrine tumors, and there has not been a systematic study of these markers with standardized immunohistochemical scoring and rigorous statistical analysis for a large series of cases (Table 3). ${ }^{14,26-29}$ This prompted us to focus on some of these factors, namely, p53 expression, cellular proliferation, the antiapoptosis markers Bcl-2 and Bcl-x, and the proapoptosis marker Bax.

Mutations of the p53 tumor suppressor gene are among the most common genetic alterations in 
Table 3 p53 and cellular proliferation in neuroendocrine tumors of the thymus

\begin{tabular}{|c|c|c|c|c|c|}
\hline Year & Author & Pathological designation & Immunochemistry & Results & Other comments \\
\hline 1996 & de Montpreville et $a l^{26}$ & Thymic neuroendocrine carcinoma & p53 & $4 / 14$ & $\begin{array}{l}\text { Strong staining in } 1 \text { cases } \\
\text { Weakly stain in } 3 \text { cases }\end{array}$ \\
\hline 1999 & Klemm et $a l^{27}$ & Pigmented carcinoid tumors & p53 & $0 / 2$ & \\
\hline 2000 & Moran et $a l^{28}$ & $\begin{array}{l}\text { Thymic neuroendocrine carcinomas } \\
\text { with combined features }\end{array}$ & p53 & $2 / 11$ & Focal positive staining \\
\hline 2001 & Goto et $a l^{29}$ & Thymic carcinoid & Ki-67 & $8 / 12$ & $0.1-6.1 \%$ labeling index \\
\hline 2003 & Tifffet et $a l^{14}$ & Thymic neuroendocrine tumors & $\begin{array}{l}\text { Ki-67 } \\
\text { p53 }\end{array}$ & $\begin{array}{l}12 / 12 \\
12 / 12\end{array}$ & $\begin{array}{l}1-52 \% \text { labeling index } \\
1-100 \%\end{array}$ \\
\hline
\end{tabular}

multistep carcinogenesis. ${ }^{30}$ In thymic tumors, p53 mutation occurs early and is associated with advanced tumor stage and biological aggressiveness. ${ }^{18,19,21,23,31-33}$ In pulmonary neuroendocrine neoplasms, p53 mutations tend to occur in more aggressive histological subtypes: it is seldom seen in low-grade tumors, such as in typical carcinoid tumor, but is more frequently evident in atypical carcinoid tumor, large-cell neuroendocrine and small-cell carcinomas. ${ }^{34-44}$ Our study showed infrequent p53 immunoreactivity in thymic neuroendocrine tumors; however, it was more common in intermediate- and high-grade than in low-grade thymic neuroendocrine tumors. This suggests that p53 gene mutations and overexpression could play a role in the pathogenesis of high-grade thymic neuroendocrine tumors, as has been shown in pulmonary neuroendocrine neoplasms.

Tumor cell growth is related to the balance between cellular proliferation and cellular loss. By using MIB-1 antibody, we found a relatively high rate of cellular proliferation in many cases irrespective of histological grade. There was a considerable range of MIB-1 staining: the mean cellular proliferation rates for low- and intermediate-grade tumors were comparable, but substantially lower than in high-grade tumors. Similarly, in the literature, thymic carcinomas and high-grade pulmonary neuroendocrine neoplasms tended to have a high rate of cellular proliferation, whereas thymomas and bronchopulmonary carcinoids had lower rates of cellular proliferation. ${ }^{17,22,23,34,37,41,43-48}$ This finding is in keeping with the observation in other thymic neoplasms that proliferative activity is linked with overexpression of p53 protein, particularly in more aggressive histologic grade tumors. ${ }^{23,25}$ However, we found that low-grade thymic neuroendocrine tumors, which morphologically resembled typical carcinoid tumors, did not have the low labeling indices that we had previously reported in their low-grade pulmonary counterparts. ${ }^{48}$ The high proliferation rate that frequently occurs in thymic neuroendocrine tumors could be a key factor in explaining its aggressive biological behavior.

Apoptosis is a complex, tightly regulated process that is frequently altered in neoplasia. ${ }^{12}$ The balance of pro- and antiapoptosis Bcl-2 family members is critical in determining if a cell undergoes apoptosis.
In thymic neuroendocrine tumors, we found a frequent but variable presence of the apoptosis markers. Overall, there was more frequent expression of the antiapoptosis markers Bcl-2 and Bcl-x, whereas the proapoptosis marker Bax was somewhat less frequently expressed. With respect to histological grade in thymic neuroendocrine tumors, we found a trend in staining that was contradictory to what has been seen in the lung, namely, that lowintermediate-grade pulmonary neuroendocrine neoplasms (carcinoid and atypical carcinoid tumors) tended to express Bax, whereas high-grade pulmonary neuroendocrine neoplasms (large-cell neuroendocrine and small-cell carcinomas) tended to express Bcl-2. ${ }^{16,38,40,49,50}$ Moreover, in the literature there are conflicting data regarding the expression of these apoptosis markers in non-neuroendocrine thymic neoplasms. ${ }^{20-24}$ Thus, the relationship of a specific apoptosis marker with histological subtype of thymic neuroendocrine tumors is often variable and inconsistent, and may not be reflective of biological behavior.

Another goal of this study was to determine if p53 expression, increased cellular proliferation, or altered apoptosis could be predictive factors of survival. In an attempt to correlate these markers with prognosis, we found that the presence of p53 in tumor cells was associated with a statistically significant decreased mean survival time (35 vs 56 months) $(P<0.05)$. This finding is not novel for thymic neoplasia, but is in keeping with the results from several other studies of non-neuroendocrine thymic neoplasms that have shown a strong correlation between p53 protein expression and survival. $^{19,23}$ However, in pulmonary neuroendocrine neoplasms there are conflicting data relating p53 expression with survival. ${ }^{16,35,36,38,39,42,43}$

With respect to apoptosis, we found a somewhat surprising finding: that the presence of an antiapoptosis marker was associated with a survival advantage. This held true in three separate statistical analyses: by the presence or absence of Bcl-x, by the use of scores in lieu of dichotomous staining, or by Bcl-x: Bax ratio scores $\geq 1$. In contrast to our findings, some prognostic factor studies of nonneuroendocrine thymic and pulmonary neuroendocrine neoplasms have shown decreased survival with the expression of another antiapoptosis marker, 
Bcl-2. ${ }^{16,24,43}$ However, there have been a few studies demonstrating that the presence of Bcl-2 in pulmonary neuroendocrine or non-neuroendocrine neoplasms was associated with better survival or that the absence of Bcl-2 was associated with aggressive behavior and poor prognosis. ${ }^{51-53}$

This disparity cannot be readily settled by locoregional differences, and it may be somewhat naive to compare thymic neuroendocrine tumors to other intrathoracic tumors. Rather, this could be explained by the small sample size in our study, as the removal of the two cases with less than 30 months survival time would shift the results into statistical nonsignificance. Other potential factors that could account for this finding include variation in histopathological classification or methodological differences inherent in immunohistochemistry (ie antibody selection, staining intensity, frequency, and scoring). It is quite conceivable that there are other factors in the apoptosis pathway up- or downstream of the Bcl-2 family that are operative. More importantly, neoplastic growth in thymic neuroendocrine tumors is not only related to the degree of necrosis and apoptosis, but to the degree of cellular proliferation. Our data seem to indicate that the enhanced cellular proliferation in thymic neuroendocrine tumors may play a stronger role than the expression of a particular apoptosis marker detected at a particular static point in time.

This study reaffirms that there are complex interactions between p53 and pro- and antiapoptosis markers that influence cellular proliferation and tumor progression in thymic neuroendocrine tumors. Overexpression of p53 tends to occur in intermediate- and high-grade tumors, but is seldom seen in low-grade tumors. There is enhanced cellular proliferation in thymic neuroendocrine tumors, despite the seemingly low-grade appearance of some tumors. The variable expression of specific pro- or antiapoptosis markers in thymic neuroendocrine tumors does not correlate well with histological grade. Our study shows that p53 expression and certain apoptosis markers correlate with survival and that the expression of these markers may account for differences in biological behavior.

\section{Acknowledgement}

We thank Diane Lawson and Deborah Sexton for assistance with immunohistochemistry.

\section{References}

1 Moran CA, Suster S. Neuroendocrine carcinomas of the thymus. Pathol Case Rev 2001;6:41-48.

2 Gal AA, Kornstein MJ, Cohen C, et al. Thymic neuroendocrine tumors: a clinicopathological and prognostic study. Ann Thorac Surg 2001;72: 1179-1182.
3 Chaer R, Massad MG, Evans A, et al. Primary thymic neuroendocrine tumors. Ann Thorac Surg 2002;74:1733-1740.

4 de Perrot M, Spiliopoulos A, Fischer S, et al. Neuroendocrine carcinoma (carcinoid) of the thymus associated with Cushing's syndrome. Ann Thorac Surg 2002;73:675-681.

5 Teh BT, Zedenius J, Kytola S, et al. Thymic carcinoids in multiple endocrine neoplasia type 1. Ann Surg 1998;228:99-105.

6 Rosai J, Levine G, Weber WR, et al. Carcinoid tumors and oat cell carcinomas of the thymus. Pathol Annu 1976;12:201-226.

7 Wick MR, Rosai J. Neuroendocrine neoplasms of the mediastinum. Semin Diagn Pathol 1991;8: $35-51$.

8 Klemm KM, Moran CA. Primary neuroendocrine carcinomas of the thymus. Semin Diagn Pathol 1999;16:32-41.

9 Moran CA, Suster S. Neuroendocrine carcinomas (carcinoid tumor) of the thymus. A clinicopathologic analysis of 80 cases. Am J Clin Pathol 2000;114: 100-110.

10 Briehl MM. Apoptosis: new ideas in pathology. In: Weinstein RS, Graham AR, Anderson RE, Benson ES, Cotran RS, Jarrett L, Wick MR, Zumwalt RE (eds). Advances in Pathology and Laboratory Medicine, Vol 9. Mosby-Year Book: St Louis, Mo 1996; pp 431-450.

11 Staunton MJ, Gaffney EF. Apoptosis: basic concepts and potential significance in human cancer. Arch Pathol Lab Med 1998;122:310-319.

12 Soini Y, Paakko P, Lehto VP. Histopathological evaluation of apoptosis in cancer. Am J Pathol 1998; 153:1041-1053.

13 Valli M, Fabris GA, Dewar A, et al. Atypical carcinoid tumour of the thymus: a study of eight cases. Histopathology 1994;24:371-375.

14 Tiffet O, Nicholson AG, Ladas G, et al. A clinicopathologic study of 12 neuroendocrine tumors arising in the thymus. Chest 2003;124:141-146.

15 Masaoka A, Monden Y, Nakahara K, et al. Follow-up study of thymomas with special reference to their clinical stages. Cancer 1981;48:2485-2492.

16 Brambilla E, Negoescu A, Gazzeri S, et al. Apoptosisrelated factors p53, Bcl2, and Bax in neuroendocrine lung tumors. Am J Pathol 1996;149:1941-1952.

17 Yang WI, Efird JT, Quintanilla-Martinez L, et al. Cell kinetic study of thymic epithelial tumors using PCNA (PC10) and Ki-67 (MIB-1) antibodies. Hum Pathol 1996;27:70-76.

18 Pich A, Chiarle R, Chiusa L, et al. p53 overexpression and thymoma prognosis. In: Marx A, Muller-Hermelink HK (eds). Epithelial Tumors of the Thymus. Plenum Press: New York 1997; pp 47-54.

19 Weirich G, Schneider P, Fellbaum C, et al. p53 alterations in thymic epithelial tumours. Virchows Arch 1997;431:17-23.

20 Tateyama H, Eimoto T, Tada T, et al. Apoptosis, bcl-2 protein, and Fas antigen in thymic epithelial tumors. Mod Pathol 1997;10:983-991.

21 Engel P, Francis D, Graem N. Expression of bcl-2 in fetal thymus, thymomas and thymic carcinomas. Association with p53 expression and review of the literature. APMIS 1998;106:449-455.

22 Pan CC, Ho DMT, Chen WYK, et al. Ki67 labelling index correlates with stage and histology but not 
significantly with prognosis in thymoma. Histopathology 1998;33:453-458.

23 Hiroshima K, Iyoda A, Toyozaki T, et al. Proliferative activity and apoptosis in thymic epithelial neoplasms. Mod Pathol 2002;15:1326-1332.

24 Chen FF, Yan JJ, Jin YT, et al. Detection of bcl-2 and p53 in thymoma: expression of bcl-2 as a reliable marker of tumor aggressiveness. Hum Pathol 1996;27:1089-1092.

25 Tomita M, Matsuzaki Y, Edagawa M, et al. Clinical and immunohistochemical study of eight cases with thymic carcinoma. BMC Surg 2002;2:3.

26 de Montpreville VT, Macchiarini P, Dulmet E. Thymic neuroendocrine carcinoma (carcinoid): a clinicopathologic study of fourteen cases. J Thorac Cardiovasc Surg 1996;111:134-141.

27 Klemm KM, Moran CA, Suster S. Pigmented thymic carcinoids: a clinicopathological and immunohistochemical study of two cases. Mod Pathol 1999;12: 946-948.

28 Moran CA, Suster S. Thymic neuroendocrine carcinomas with combined features ranging from well-differentiated (carcinoid) to small cell carcinoma. A clinicopathologic and immunohistochemical study of 11 cases. Am J Clin Pathol 2000;113:345-350.

29 Goto K, Kodama T, Matsuno Y, et al. Clinicopathologic and DNA cytometric analysis of carcinoid tumors of the thymus. Mod Pathol 2001;14:985-994.

30 Sarkiss M, Hsu B, el-Naggar AK, et al. The clinical relevance and assessment of apoptotic cell death. Adv Anat Pathol 1996;3:205-211.

31 Tateyama H, Eimoto T, Tada T, et al. p53 protein expression and p53 gene mutation in thymic epithelial tumors. An immunohistochemical and DNA sequencing study. Am J Clin Pathol 1995;104:375-381.

32 Hayashi Y, Ishii N, Obayashi C, et al. Thymoma: tumour type related to expression of epidermal growth factor (EGF), EGF-receptor, p53, v-erb B and ras p21. Virchows Arch 1995;426:43-50.

33 Hino N, Kondo K, Miyoshi T, et al. High frequency of p53 protein expression in thymic carcinoma but not in thymoma. Br J Cancer 1997;76:1361-1366.

34 Barbareschi M, Girlando S, Mauri FA, et al. Tumour suppressor gene products, proliferation, and differentiation markers in lung neuroendocrine neoplasms. J Pathol 1992;166:343-350.

35 Roncalli M, Doglioni C, Springall DR, et al. Abnormal p53 expression in lung neuroendocrine tumors. Diagnostic and prognostic implications. Diagn Mol Pathol 1992;1:129-135.

36 Lohmann D, Putz B, Reich U, et al. Mutational spectrum of the p53 gene in human small-cell lung cancer and relationship to clinicopathological data. Am J Pathol 1993;142:907-915.

37 Rusch VW, Klimstra DS, Venkatraman ES. Molecular markers help characterize neuroendocrine lung tumors. Ann Thorac Surg 1996;62:798-809.

38 Coppola D, Clarke M, Landreneau R, et al. Bcl-2, p53, CD44, and CD44v6 isoform expression in neuroendocrine tumors of the lung. Mod Pathol 1996;9:484-490.
39 Przygodzki RM, Finkelstein SD, Langer JC, et al. Analysis of p53, K-ras-2, and C-raf-1 in pulmonary neuroendocrine tumors: correlation with histological subtype and clinical outcome. Am J Pathol 1996;148:1531-1541.

40 Wang DG, Johnston CF, Sloan JM, et al. Expression of Bcl-2 in lung neuroendocrine tumours: comparison with p53. J Pathol 1998;184:247-251.

41 Zirbes TK, Lorenzen J, Baldus SE, et al. Apoptosis and expression of bcl-2 protein are inverse factors influencing tumour cell turnover in primary carcinoid tumours of the lung. Histopathology 1998;33: 123-128.

42 Onuki N, Wistuba II, Travis WD, et al. Genetic changes in the spectrum of neuroendocrine lung tumors. Cancer 1999;85:600-607.

43 Santinelli A, Ranaldi R, Baccarini M, et al. Ploidy, proliferative activity, p53 and bcl-2 expression in bronchopulmonary carcinoids: relationship with prognosis. Pathol Res Pract 1999;195:467-474.

44 Laitinen KL, Soini Y, Mattila J, et al. Atypical bronchopulmonary carcinoids show a tendency toward increased apoptotic and proliferative activity. Cancer 2000;88:1590-1598.

45 Costes V, Marty-Ane C, Picot MC, et al. Typical and atypical broncho-pulmonary carcinoid tumors: a clinicopathologic and Ki-67 labeling study. Hum Pathol 1995;26:740-745.

46 Durham JR, Nakhleh RE, Swanson PE, et al. Proliferation markers MIB-1 and PCNA in pulmonary neuroendocrine tumors. Appl Immunochem 1995; 3:174-183.

47 Bohm J, Koch S, Gais P, et al. Prognostic value of MIB-1 in neuroendocrine tumours of the lung. J Pathol 1996;178:402-409.

48 Arbiser ZK, Arbiser JL, Cohen C, et al. Neuroendocrine lung tumors: grade correlates with proliferation but not angiogenesis. Mod Pathol 2001; 14:1195-1199.

49 Jiang SX, Kameya $\mathrm{T}$, Sato $\mathrm{Y}$, et al. Bcl-2 protein expression in lung cancer and close correlation with neuroendocrine differentiation. Am J Pathol 1996; 148:837-846.

50 Pal'tsev MA, Demura SA, Kogan EA, et al. Role of Bcl2, Bax, and Bak in spontaneous apoptosis and proliferation in neuroendocrine lung tumors: immunohistochemical study. Bull Exp Biol Med 2000; 130:697-700.

51 Eerola AK, Ruokolainen H, Soini Y, et al. Accelerated apoptosis and low bcl-2 expression associated with neuroendocrine differentiation predict shortened survival in operated large cell carcinoma of the lung. Pathol Oncol Res 1999;5:179-186.

52 Laudanski J, Chyczewski L, Niklinska WE, et al. Expression of bcl-2 protein in non-small cell lung cancer: correlation with clinicopathology and patient survival. Neoplasma 1999;46:25-30.

53 Ohmura Y, Aoe M, Andou A, et al. Telomerase activity and Bcl-2 expression in non-small cell lung cancer. Clin Cancer Res 2000;6:2980-2987. 